\title{
Le fard des couleurs de rhétorique à la Renaissance
}

\section{Adeline Desbois-lentile}

\section{OpenEdition}

Journals

Édition électronique

URL : http://journals.openedition.org/rhetorique/1033

DOI : $10.4000 /$ rhetorique. 1033

ISSN : 2270-6909

\section{Éditeur}

UGA Éditions/Université Grenoble Alpes

\section{Édition imprimée}

ISBN : 978-2-37747-248-2

\section{Référence électronique}

Adeline Desbois-lentile, "Le fard des couleurs de rhétorique à la Renaissance », Exercices de rhétorique [En ligne], 15 | 2020, mis en ligne le 07 novembre 2020, consulté le 10 décembre 2020. URL : http:// journals.openedition.org/rhetorique/1033; DOI : https://doi.org/10.4000/rhetorique.1033

Ce document a été généré automatiquement le 10 décembre 2020.

\section{(c) (i) (2)(2)}

Les contenus de la revue Exercices de rhétorique sont mis à disposition selon les termes de la Licence Creative Commons Attribution - Pas d'Utilisation Commerciale - Partage dans les Mêmes Conditions 4.0 International. 


\title{
Le fard des couleurs de rhétorique à la Renaissance
}

\author{
Adeline Desbois-lentile
}

1 Au début de sa Légende des Vénitiens, texte pamphlétaire dénigrant la Sérénissime et avec elle la papauté qui en était l'alliée, l'historien et poète Lemaire de Belges prévient son lecteur qu'il lui plaise « supporter benignement la grosse tournure du langaige peu elegant " à l'œuvre dans le texte. Les épîtres dédicatoires et la fin des prologues sont le lieu privilégié de telles déclarations, et on n'aurait là qu'un simple topos de modestie, captatio beneuolentiae à laquelle recourent les écrivains pour s'attirer les bonnes grâces de leur auditoire, si Lemaire n'ajoutait pas aussitôt : « car j'ay plus eu de regart a ce que la narration historialle soit garnie de verité que coulouree de fleurs de rhetoricque ${ }^{1}$.» La reconnaissance de la " grosse tournure du langaige peu elegant » ne serait donc pas l'aveu (réel ou feint) d'une faiblesse de la part d'un écrivain en peine de recourir à un meilleur style, mais la revendication du seul style possible eu égard au contenu historiographique de l'œuvre. Un contemporain de Lemaire, Jean Bouchet, expose la même idée au début de ses Annales d'Aquitaine, suppliant ses lecteurs qu'il leur " plaise excuser et supporter l'imperfection du langage vulgaire, a l'ornement et acoustrement duquel [il ] n'[a] prins peine ne labeur : mais seulement a la verité de l'histoire ${ }^{2}$ ». Et Jean de Léry, trois décennies plus tard, assure encore à «ceux qui aiment mieux la vérité dite simplement que le mensonge orné et fardé de beau langage ${ }^{3}$ » qu'ils trouveront de l'intérêt à son Histoire d'un voyage fait en la terre du Brésil.

2 Ce constat d'une contradiction entre les ornements de la rhétorique et l'expression de la vérité est aussi tenace qu'il est vague et peut concerner autant le plan de l'inuentio que celui de l'elocutio, comme on le voit sous la plume de Du Haillan, qui revendique auprès du roi et de ses lecteurs la vérité de son Histoire de France :

[...] ceux qui escrivent l'Histoire de leur temps sont agitez de diverses passions, et sont contraints ou de mentir appertement s'ils louent en tout et par tout leurs Princes ou s'ils favorisent leur nation, ou si en tout ils blasment leurs ennemis, ou de dissimuler ou de pallier la verité, ou de bigarrer les choses, ou de farder et dorer de belles parolles leurs escrits et les actions de leursdits Princes ${ }^{4}$. 
Le binôme "leurs escrits et les actions" met en évidence une caractéristique de l'écriture historique : les mots ("leurs escrits») sont en prise directe avec les choses ( (les actions»). Introduire volontairement dans le récit historique des propos ne renvoyant pas à ce qui a été ou aurait pu être, c'est donc chercher à modifier le passé lui-même. Un exemple qu'aurait sans doute pu choisir Du Haillan est celui de la généalogie troyenne des princes français, qu'il s'attache longuement à récuser contre ceux qu'il appelle les historiens " courtisans ", plus soucieux de flatterie que de vérité.

3 Si le reproche de Du Haillan ne vise pas tant les figures de style que les faits, le propos de Lemaire de Belges, Bouchet ou Léry, en revanche, concerne plus directement les caractéristiques de l'écriture historique ou documentaire: une "grosse tournure " marquée par l'« imperfection » et une manière de parler "simplement ». Derrière ces formules, croyons-nous, se cache le rejet de certains procédés, certains tropes en particulier aptes aussi bien à orner qu'à induire de la fausseté. Elles invitent ainsi à analyser la place des "figures de style», comme on les appelle aujourd'hui, dans l'écriture de la vérité à la Renaissance.

\section{Des métaphores contre l'éloquence}

Renoncer à l'élégance au nom de la vérité est un lieu commun sous la plume d'auteurs revendiquant la vérité de leurs écrits, quel que soit le genre choisi. Dans une mise en abyme au seuil de son Heptaméron, Marguerite de Navarre fait dire à Parlamente que le dauphin de France aurait eu l'ambition d'écrire un recueil de nouvelles avec d'autres compagnons, à l'exclusion des "gens de lettres", "de peur que la beauté de rhetoricque feist tort en quelque partie à la verité de l'histoire ${ }^{5}$ », tandis que Bernard Palissy affirme de son côté dans l'épître dédicatoire ouvrant ses traités sur l'agriculture, la science des jardins et des villes fortes: "J'aime mieux dire verité en mon langage rustique, que mensonge en un langage rhethorique ${ }^{6}$.»

5 Le discours déployé pour critiquer les ornements de la rhétorique est volontiers métaphorique, et recourt aux images mêmes utilisées par ses défenseurs: la valeur axiologique des «fleurs" et des «couleurs", désormais suspects de n'être que des ornements trompeurs, est inversée, comme l'a montré Marie-Hélène Prat à propos d'Agrippa d'Aubigné ${ }^{7}$. Le rejet du beau langage repose sur ces deux images, autorisant chacune un déploiement métaphorique secondaire. Celle des "fleurs de rhétorique " réduit la "rhétorique » à un exercice de style, pour opposer la beauté éphémère de la langue d'une part, à l'enseignement durable ou fruit qu'on peut retirer de la lecture de l'autre. Le médecin et chirurgien Pierre Hassard recourt à cette opposition dans un de ces prologues à valeur de captatio beneuolentiae qui ouvre sa traduction de la Chirurgie de Paracelse :

Je sçay Monseigneur que vostre Excellence ne trouvera icy grande elegance ne ornement de langaige, mais j'ay esté contrainct pour la proprieté et grandeur de ceste science d'user d'un stil bas et rude, sans aucune affectation, car en toutes choses le fruict se doibt plus priser que la fleur'.

Là encore, pour Pierre Hassard, le recours à un « stil bas et rude » est présenté comme une nécessité. L'auteur revient sur cette question du style dans le prologue qu'il adresse cette fois-ci aux lecteurs, en exprimant l'idée d'une incompatibilité entre "rhétorique » et « médecine », le seconde devant avoir la priorité sur la première : 
Jaçoit que le stil te semblera rude et simple, la cause est pource que la Medecine n'est point rethorique, et par ainsi aussi la chose ira avant, et aussi la Medecine ira la droicte voye, tout ainsi comme le potier faict son pot $^{9}$.

En affirmant que "la chose ira avant», Pierre Hassard ne fait pas qu'énoncer la prédominance de la médecine sur la rhétorique. Il fait aussi du savoir médical un objet préexistant à tout discours, une "chose » à laquelle la rhétorique comme art de la parole ne peut être qu'extérieure.

On retrouve là le second réseau métaphorique, plus fréquent dans le corpus étudié ici : celui de la couleur, associée au mensonge, si bien que le terme semble acquérir une connotation péjorative durable ${ }^{10}$. La vérité, en revanche, n'est pas associée à la transparence (idée qu'on ne trouve nulle part) mais figure comme un objet que la couleur menace de transfigurer, ou de défigurer. L'expression «couleurs de rhétorique » n'est pas nouvelle ${ }^{11}$ mais remotivée sémantiquement par les auteurs. Dans un syncrétisme des arts, l'art oratoire est associé à l'art visuel et à la peinture en particulier, art de la couleur. Dans son Traité de science poétique, Jean Thenaud présente ainsi Hermafroditus, fils du dieu de l'éloquence Mercure et de la déesse de la beauté Aphrodite, comme l'allégorie de " paincture de rhetoricque laquelle est plus delectable que profitable, et est engendrée de superfluité, non de raison et verité ». Et Thenaud ajoute aussitôt :

Par ce faingnent les poetes que Sophie ne voulut estre mariée à Mercure, car combien que eloquence decore moult la personne, touttesfoys sapience refuse le superflu et trop exquis parler ${ }^{12}$.

La rhétorique comme "couleur" appartient au domaine des apparences, volontiers futile voire dangereux, par opposition à ce qui est perçu comme le fondement de la vérité et du savoir. L'antithèse présentée par Lemaire entre "garnie de verité » et «coulouree de fleurs de rhetoricque ${ }^{13}$ » ou "enrichie d'aucunes couleurs d'art oratoire $^{14} »$ met en évidence la différence de statut entre la vérité comme matière et garnissage, et la rhétorique comme coloration secondaire. Non seulement les couleurs sont décoration et ornement, comme si une pensée pouvait totalement être dissociée de la forme qui l'exprime, mais à ce titre, elles sont également un voile risquant de masquer la vérité.

7 Picturales, les couleurs de rhétorique sont aussi celles du maquillage comme le révèle l'emploi du terme fard en contexte métalinguistique, à connotation toujours péjorative, à l'image de Léry critiquant « le mensonge orné et fardé de beau langage ${ }^{15}$ ». Ce refus du fard est particulièrement présent sous la plume des théologiens, Calvin voyant dans un propos « nud sans aucun fard ${ }^{16}$ » tenu par Isaïe la preuve de son caractère prophétique, pendant que le prédicateur René Benoist assure que la vérité ne doit pas être «fardée ou enrichie d'un beau et élégant parler ${ }^{17} »$. Dans les textes, couleur et fard, colorer et farder apparaissent au sein des mêmes syntagmes quand ils ne forment pas un binôme synonymique, d'un bout à l'autre du siècle et dans des ouvrages de facture différente : Molinet regrette ainsi que des faits banals aient trouvé "picteurs amoureux des images » et été ainsi «fardéz de couleurs ${ }^{18}$ ", tandis que Noël Du Fail affirme qu' « il n'y avoit fard ne couleur de bien dire, fors une pure verité » dans les propos rustiques qu'il reproduirait $^{19}$, et que Robert Estienne critique les «langages tant fardés et colorés par les hommes ${ }^{20} »$. Montaigne encore loue l'éloquence de Socrate, y voyant paradoxalement l'ornement dans le naturel, contre les artifices de la rhétorique. On ne pouvait donc attendre de Socrate un discours oratoire pour se défendre de sa condamnation à mort : 
sa riche et puissante nature, eust elle commis à l'art sa defense : et en son plus haut essay, renoncé à la verité et naïveté, ornemens de son parler, pour se parer du fard, des figures et feintes, d'une oraison apprinse ${ }^{21}$ ?

Montaigne, toutefois, ne peut résister au plaisir de la rhétorique, modelant sa phrase sur les figures du nombre, par des effets de rythme et le retour de sonorités, jusque aux paronomastiques "fard, figures et feintes ", preuve que les figures, même dénigrées, conservent un certain attrait, voire une certaine efficacité. C'est là tout le paradoxe de la critique de l'ornement, chez des auteurs à la prose parfois fortement travaillée. La question qui se pose dès lors est de savoir quelles sont les figures de style qui, à la Renaissance, apparaissent comme plus propres à farder le discours que les autres.

\section{Les formes du style simple}

La condamnation éthique de l'ornement, suspect d'embellissement et de travestissement, participe de la réflexion sur les genres d'écrire, dans la tradition rhétorique héritée de Cicéron et Quintilien selon laquelle il existe une tripartition stylistique entre style haut, moyen et bas, chaque style ayant ses propres procédés et ses visées. Ce qui se joue dans la critique des « couleurs » et du « fard » de la rhétorique, et la revendication d'une vérité dite "simplement», c'est alors l'élection (au moins dans le discours, si ce n'est dans les faits) du style simple contre les autres. Le style simple, en effet, se caractérise par une ornementation minimale que Cicéron rapprochait déjà dans une longue métaphore filée de ces femmes qui plaisent sans apprêt et sans fard ${ }^{22}$. Cicéron recommande au contraire à l'orateur de style simple de fuir tout ce qui s'éloigne trop de l'usage, comme les néologismes ou les archaïsmes, d'être parcimonieux dans les métaphores (à l'exception des plus faciles), les répétitions marquées, les allégories recherchées, ou les prosopopées, et de déployer de ce fait un style en sourdine ${ }^{23}$. Le style simple se situe du côté de la conversation familière, contre toute forme d'affectation. Cela ne signifie pas que le style simple soit dépourvu de toute figure, et Montaigne observe au contraire dans son essai consacré à la vanité des paroles que tout discours, même le plus spontané, recourt aux couleurs de rhétorique et que ce sont les noms mêmes qui leur sont donnés qui en renforcent pompeusement le caractère artificiel :

Oyez dire metonymie, metaphore, allegorie, et autres tels noms de la grammaire, semble-il pas qu'on signifie quelque forme de langage rare et pellegrin? ce sont titres qui touchent le babil de vostre chambriere ${ }^{24}$.

Il n'en reste pas moins que l'opposition joue fortement dans l'imaginaire des styles, et ce qui est en jeu est le recours à un style volontairement figural, perçu comme artificiel, contre un style naturel non figural. L'expression de la vérité doit se passer de couleurs trop voyantes.

9 Ces questions prennent un relief particulier lorsqu'il n'est plus seulement question de vérité historique ou factuelle, mais divine. Calvin argumente dans le sens d'une efficacité du style simple qui, même dépourvu d'ornements rhétoriques et même sans avoir « une telle apparence que peuvent desirer gens lettrez ${ }^{25}$ » selon ses mots, repose au contraire sur le pouvoir que lui confère la vérité elle-même :

[...] si la doctrine de s. Jehan ou s. Paul estoit bien parée de couleurs de Demosthene ou Ciceron, possible qu'elle auroit plus de grace pour alleicher à soy les lecteurs, mais il ne resteroit point la centieme partie de ceste gravité que nous y sentons pour toucher les consciences au vif, et de la majesté pour authoriser tout ce qui est là contenu ${ }^{26}$. 
Du côté catholique, René Benoist ne dit pas autre chose :

Si nostre diction n'est assés propre ou elegante, ou nos sentences non assez bien ageancées, nous disons avec sainct Paul, que le royaume de Dieu n'est en la parole : et que le parler elegant n'est requis à la verité, laquelle est assez belle, excellente et desirable d'elle-mesme, sans estre fardee ou enrichie d'un beau et elegant parler, lequel est necessaire aux heretiques, pour persuader leur mensonge, et farder et colorer leur vieille flasque, et ridee masque d'heresie ${ }^{27}$.

On retrouve dans ces affirmations la position de certains historiens : la vérité n'a pas besoin de l'élégance des couleurs car elle produit un discours efficace de lui-même ; bien au contraire, les couleurs sont suspectes d'affaiblir le fondement véridique du propos. Dans une tradition remontant cette fois-ci à saint Augustin $^{28}$, le style simple devient la langue de la révélation, et les auteurs élaborent une opposition entre le style simple divin et l'élégance artificielle de la langue des hommes ${ }^{29}$ qui se poursuit à l'âge classique $^{30}$. La valeur de l'opposition entre style simple et style figuré se renverse : si le style simple est perçu comme nécessaire, mais bas ou rude par les historiens, il est au contraire pensé par les théologiens comme le langage de la révélation qui tire sa force de lui-même sans avoir besoin du soutien de l'art rhétorique. Les fleurs et couleurs de rhétorique, auxquelles Lemaire ou Bouchet semblent renoncer à regret, ne sont plus qu'un vain artifice, outil de la faiblesse humaine.

\section{Les tropes, figures de la fiction}

11 Que l'éloquence soit porteuse de fausseté : le lieu commun pourrait bien reposer sur des caractéristiques internes aux « couleurs » de rhétorique elles-mêmes. Les textes, on l'a $\mathrm{vu}$, restent vagues dans leurs critiques et se borne à y voir une forme de mensonge. Il n'est peut-être pas impossible, toutefois, d'identifier des procédés qui feraient plus tort à la vérité que les autres. Il semble utile de distinguer sur ce point figures et tropes, auxquels Quintilien consacre deux livres différents et qu'il définit de la façon suivante : la figure " consiste à donner au langage une forme éloignée de l'expression commune et spontanée " (conformatio quaedam orationis remota a communi et primum se offerente ratione), tandis que le trope est «le transfert d'une expression de sa signification naturelle et principale à une autre, afin d'orner le style » (" sermo a naturali et principali significatione tralatus ad aliam ornandae orationis gratia $\left.{ }^{31} »\right)$. De ce fait, les figures seraient artificielles par l'écart qu'elles suscitent vis-à-vis d'autres formes d'expression plus communes, elles introduiraient une forme d'opacité dans l'énoncé en attirant l'attention sur la forme, en donnant de l'éclat au propos, mais elles n'affectent pas le statut ontologique de l'énoncé. Le trope en revanche permet une double lecture, littérale et figurée, qui est en elle-même porteuse de fausseté puisque le sens figuré est juste mais faux littéralement. Autrement dit, le vice associé aux figures est celui de l'affectation, tandis que le trope, parce qu'il implique un véritable transfert du sens littéral au sens figuré, peut confiner au mensonge.

12 Ces observations ne sont pas explicitées dans les textes mais il nous semble que les tropes sont plus particulièrement visés par la critique d'une éloquence colorée à la Renaissance, et que l'enjeu n'est pas seulement celui du clinquant. Le problème posé par la lecture des tropes est mis en scène par Rabelais dans ce passage où frère Jean doit rendre compte de sa liberté de parole :

- Ainsi, dist le moyne, à ces diables : ce pendent qu'ilz durent.

«Vertus dieu qu'en eust faict ce boyteux? 
« Le cor dieu il prent plus de plaisir quand on luy faict present d'un bon couble de beufz.

- Comment (dist Ponocrates), vous jurez frere Jean?

- Ce n'est (dist le moyne) que pour orner mon langaige. Ce sont couleurs de rhetorique Ciceroniane ${ }^{32}$.

La question de Ponocrates sous-entend une accusation de blasphème : les jurons de frère Jean reposent en effet sur des métaphores anthropomorphisant Dieu à qui on prête vertu («Vertus Dieu») et corps («Le cor Dieu») si on en fait une lecture littérale. La défense de frère Jean consiste au contraire à réduire le juron à un simple ornement, « couleur » qui ne doit pas être interprétée littéralement. Il en fait une simple figure, là où Ponocrates y voit un possible dédoublement du sens.

Il semble que les auteurs de la Renaissance aient été sensibles à ce qu'on peut appeler les figures de la fiction ${ }^{33}$, c'est-à-dire le fait que certains procédés, les tropes en particulier, soient en eux-mêmes porteurs de fictionnalité. De la liste des principaux tropes relevés par Quintilien (métaphore, synecdoque, métonymie, antonomase, onomatopée, épithète rhétorique, allégorie, périphrase, hyperbole), c'est l'hyperbole qui est nommée par Montaigne dans une réflexion portant sur la vérité et le mensonge :

Moy-mesme, qui fais singuliere conscience de mentir : et qui ne me soucie guiere de donner creance et authorité à ce que je dis, m'apperçoy toutesfois, aux propos que j'ay en main, qu'estant eschauffé ou par la resistance d'un autre, ou par la propre chaleur de ma narration, je grossis et enfle mon subject, par voix, mouvemens, vigueur et force de parolles : et encore par extention et amplification: non sans interest de la verité nayfve : Mais je le fais en condition pourtant, qu'au premier qui me rameine, et qui me demande la verité nue et crue : je quitte soudain mon effort, et la luy donne, sans exaggeration, sans emphase, et remplissage. La parole vive et bruyante, comme est la mienne ordinaire, s'emporte volontiers à l'hyperbole ${ }^{34}$.

Le jeu des accumulations donne à ce passage une allure hyperbolique qui exemplifie ce que Montaigne avoue de son propre goût pour les hyperboles. Le philosophe met en évidence les limites posées par l'emploi du trope, qui ne doit pas faire perdre de vue la vérité. À l'hyperbole s'oppose « la verité nayfve ", dite encore « la verité nue et crue » : même si les travaux sur Montaigne ont pu mettre en évidence l'efficacité pragmatique du trope au-delà de sa fausseté littérale ${ }^{35}$, la déformation impliquée par le trope pose visiblement problème à un auteur qui refuse par ailleurs le mensonge.

C'est bien ce même trope dont Molinet semble critiquer l'usage, pour sa capacité à transformer les faits du passé :

Aultres exploicts de petite value, capable de basse renommée, sont assis en hault game, pour avoir trouvé picteurs amoureux des images, lesquelz ilz ont fardéz de couleurs apparentes pour avoir gloire entre les preux. Hercules, le robuste poing de la Grèce, le dompteur des ravissants larrons et le fourdroyeur des terribles monstres sauvaiges, doibt richement guerdonner Omère et aultres picteurs qui, par leurs poesies et versifications heroiques, ont augmenté sa vaillance jusques à soubtenir les cieux. Aeneas, le chevalier aventureu, fuytif de Troye, de qui descendit la très sacrée semence imperialle, fut bien heureu que Virgille le poète le prist en sa grace ; car, en faveur et contemplation des Romains, le extolla par dessus les nues, et toutesfois aucuns luy ont imparti note de prodition ${ }^{36}$.

Molinet multiplie dans ce passage les hyperboles pour dénoncer les «picteurs amoureux des images ", responsables d'une falsification de l'histoire. Il s'agit en l'occurrence de deux poètes, Homère et Virgile, si bien que le vocabulaire pictural semble signaler la valeur artistique de ces récits (ut pictura poesis), à l'opposé d'une prose qui dirait la vérité « au naturel ». Même si la prose des Chroniques n'est pas tout à fait exempte par endroits de semblables effets de style ${ }^{37}$, Molinet semble placer ces 
récits poétiques du côté du faux, en raison même de leur emploi des hyperboles source d'un renversement, selon lui, du sens de l'histoire.

Ainsi les tropes, non seulement, donnent de l'éclat au discours, comme l'affirmait Quintilien, mais sont en eux-mêmes porteurs de fausseté. L'écriture historique, à l'inverse, nécessiterait le recours au sens propre, garant de l'expression de la vérité. De fait, Jacques Peletier observe dans son Art poétique, au sujet des métaphores : «Et quasi n'y a figure plus fréquente en toutes sortes d'auteurs, fors par aventure ès historiens,

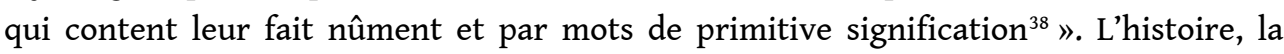
grande, peut se dire sans élégance parce qu'elle a la vérité pour elle. Cette observation se vérifie chez des auteurs pouvant se targuer d'être aussi bien poètes qu'historiens, comme d'Aubigné, dont Marie-Hélène Prat a montré que son Histoire universelle était moins riche en métaphores et métonymies que Le Printemps et Les Tragiques, et que les images s'y concentraient dans le paratexte (préfaces et conclusions des différents livres $^{39}$ ), ou Lemaire de Belges, qui ne recourt pas aux mêmes procédés stylistiques selon la nature de ses textes. De fait, si Lemaire reconnaît, dans ses œuvres à substrat historique, recourir à une " grosse tournure du langaige peu elegant ", les tropes y sont rares. Malgré la teneur pamphlétaire du propos, on ne relève guère, dans La Legende des Venitiens, qu'une métaphore lexicalisée (« ilz n'ont acoustumé de pescher synon en eaue trouble ${ }^{40} »$ ) de laquelle on peut rapprocher une comparaison («a l'exemple du bon laboureur qui coppe une mauvaise branche pour faire pulluler les autres $\left.{ }^{41} »\right)$, et quelques "invectives exclamatoires ${ }^{42}$ ", fictions de discours se combinant bien souvent à ces autres tropes que sont l'hyperbole, la métaphore ou la personnification ( $\mathrm{O}$ mauldicte, infernalle avarice [...]», «O precurseurs d'antechrist et procureurs de Mahomet! N'estes vous pas dignes d'estre venuz la ou vous estes $\left.{ }^{43} »\right)$. Ou encore, filant dans la péroraison, plus oratoire, la syllepse sur le nom de Marguerite d'Autriche :

0 , si vous eussiez sceu finer en voz tresors de une semblable vive perle pacifique, de une telle margarite australle, et de progeniture auguste, vous estiez a tousjours riches! Mais non, car vostre trop grant saigesse est tournee en folie, et vostre fastidieuse gloire en ignominie ${ }^{44}$.

Dans son Traicté de la différence des schismes et des conciles de l'église, tout aussi pamphlétaire, Lemaire recourt à nouveau à l'invective ( $O$ faulse et malicieuse pharisienne hypocrisie sacerdotall $\left.{ }^{45}[. .] ».\right)$, mais la place des tropes y est encore plus limitée; Lemaire y préfère les comparaisons aux métaphores ${ }^{46}$, ces dernières n'étant introduites qu'à travers des proverbes :

Ceste matiere des scismes et divisions est si confuse et si odieuse que je la voudroie bien passer soubz silence, se ce n'estoit pour venir à quelque bonne fin. Car bien souvent advient que qui veult aler en ung vergier plaisant, il faut passer par voyes estroictes et fangeuses, et fault cueillir les roses entre les espines et le grain en la paille ${ }^{47}$.

La moisson est tout aussi maigre dans l'Histoire du prince Sophy, le Blason des armes des Venitiens ou le Traité des pompes funeralles. Lemaire pourtant manie facilement l'allégorie et avec elle l'ensemble des autres tropes, mais les réserve semble-t-il aux textes mettant en scène des personnages ou des épisodes fictionnels. Le phénomène est net dans les composites Illustrations de Gaule et singularitez de Troye, où l'on observe, comme je l'ai montré ailleurs, que la répartition des différents procédés rhétoriques et stylistiques varie selon le degré d'historicité, et inversement de fictionnalité, du passage ${ }^{48}$. En ce qui concerne les figures et tropes en particulier, la prose historique des Illustrations est ainsi celle qui est la plus dépourvue de figuralité : l'usage du lexique 
privilégie le sens propre, à l'exception de quelques catachrèses ; les figures dominantes, de répétition et d'accumulation, restent peu saillantes et ressortissent avant tout à un usage formulaire. À l'inverse, la partie centrale appelée "roman de Pâris ", qui met en scène les dieux et déesses de l'olympe, multiplie les tropes, comme si au statut fictif des personnages devait correspondre des procédés de dédoublement qui, lus au sens littéral, créent une forme de fiction, comme si le processus d'interprétation allégorique mis en place pour les personnages devait se refléter dans des procédés appelant un sens figuré. Parmi les personnages allégoriques, la déesse Vénus est celle qui recourt le plus massivement aux tropes ${ }^{49}$, dans le discours qu'elle tient devant le juge Pâris en charge d'octroyer la pomme d'or à la plus belle des déesses : autant la paronomase entre couleurs et douleurs annonçait dans le Temple d'honneur et de vertus un prosimètre allégorique à valeur encomiastique ${ }^{50}$, autant dans le discours de Vénus, il révèle le danger des tropes, lorsque la rupture entre l'éthique et l'esthétique a été consommée ${ }^{51}$ et que le discours sert d'autres fins que la vérité :

L'eloquence artificielle de dame Venus, ses paroles delicates, et sa douce persuasion causerent telle efficace et telle emotion au coeur du jeune adolescent Paris, que encores en pourra il maudire les rhetoriques couleurs, qui luy seront retorquees en douleurs ${ }^{52}$.

L'affinité des tropes avec la fiction (même lorsque celle-ci est au service d'un discours au fondement véridique) est également confirmée, semble-t-il, par la Concorde des deux langages dont la structure en diptyque met en évidence deux modèles d'écriture: la terza rima du temple de Vénus, associée à la poésie italienne et courtoise, multiplie les tropes, à l'inverse du temple de Minerve, recourant à l'alexandrin et la prose, et associé à la littérature historique et morale ${ }^{53}$.

17 En matière de théologie, toutefois, face au discours par endroits fortement imagé des Écritures, se pose la question de savoir à quelles conditions un discours peut être véridique et figuré. Olivier Millet observe ainsi une méfiance, chez Calvin, vis-à-vis de l'ornement poétique ${ }^{54}$, sauf dans les passages

où le style poétique exprime le vrai quand il est incroyable, par exemple la prophétie christologique, ou quand il est le seul apte à exprimer les objets ou l'expérience de la foi ; bref, quand les figures sont à prendre au pied de la lettre.

Dans ces cas-là, il n'y a pas de risque d'" excès » ou de « fiction " ${ }^{55}$.

De ce fait, le reproche de fausseté que l'on peut exprimer à l'encontre des tropes tombe dès lors qu'une lecture littérale est possible parallèlement à la lecture figurée mais il s'agit là de cas singuliers. L'impératif sous-jacent est celui de la convenance : de même que dans les Illustrations de Lemaire, les tropes apparaissent comme le procédé le plus apte à mettre en scène les dieux de l'olympe, leur emploi est ici légitimé pour évoquer le fonctionnement très spécifique du texte biblique.

On s'éloigne, toutefois, dans ces deux cas, de la vérité historique ou scientifique des hommes, contrainte si l'on en croit les auteurs à être énoncée en style bas, simple, rude - autant de parasynonymes qui masquent, derrière la critique d'une éloquence trop ornée, la revendication du sérieux de telle histoire ou de tel traité. Si l'emploi des tropes dans les textes peut répondre à un impératif d'ordre pragmatique ou présenter une valeur cognitive, ils restent pensés comme peu compatibles avec le discours véridique. Là où la figure peut créer de l'opacité en attirant l'attention sur la forme du texte, le trope, par le dédoublement du sens, crée de l'incertitude, et ouvre une brèche interprétative. Ce sont eux, plus que les figures, qui à l'échelle microstructurale 
peuvent être entachés du soupçon de mensonge : de fait, les tropes mentent toujours, littéralement.

\section{NOTES}

1. J. Lemaire de Belges, La Légende des Vénitiens (1509), éd. A. Schoysman, Bruxelles, Académie royale de Belgique, 1999, prologue, p. 5.

2. J. Bouchet, Les Annales d'Aquitaine, Poitiers, Enguilbert de Marnef et Jacques Bouchet, 1524, prologue, n. p.

3. J. de Léry, Histoire d'un voyage fait en la terre du Brésil (1557), éd. F. Lestringant, Montpellier, M. Chaleil, 1992, p. 34.

4. B. de Girard Du Haillan, L'Histoire de France, Paris, P. L'Huillier, 1576, « Preface aux lecteurs », n. p.

5. M. de Navarre, L'Heptaméron, éd. N. Cazauran, Paris, Gallimard, 2000, p. 66.

6. B. Palissy, Recepte veritable, par laquelle tous les hommes de la France pourront apprendre a multiplier et augmenter leurs thresors, La Rochelle, B. Berthon, 1573, épître dédicatoire «A monseigneur le mareschal de Montmorancy», n. p. Palissy évoque à nouveau son «langage rustique, et mal orné » puis « rustique et mal poli » dans l'épître aux lecteurs (n. p.).

7. M.-H. Prat, «Les "fleurs" et les "couleurs": sur quelques métaphores de l'écriture dans les Tragiques d'Agrippa d'Aubigné ", dans O. Pot dir., Poétiques d'Aubigné, Genève, Droz, 1999, p. 79-93.

8. La grande, vraye, et parfaicte chirurgie, du tresdocte et tresçavant Prince de Philosophie et de Medecine [...] Paracelse [...] nouvellement traduicts en langue Françoise, par M. Pierre Hassard, Anvers, Guillaume Silvius, 1567, épître dédicatoire "A hault et puissant prince, messire Lamoral d'Egmont », n. p. V. Worth-Stylianou a observé que plusieurs médecins rejettent explicitement un «langage fardé » ou "beau style », et elle mentionne aux côtés de P. Hassard le traducteur anonyme de L'Anatomie des nerfs (1556) (voir Histoire des traductions en langue française. $\mathrm{XV}^{e}$ et $\mathrm{XVI} I^{e}$ siècles, dir. V. Duché, Lagrasse, Verdier, 2015, p. 765).

9. La grande, vraye, et parfaicte chirurgie, op. cit., f. 3 r.

10. Le Dictionnaire de l'Académie française de 1694 définit le terme couleur de la façon suivante (Paris, Veuve Jean-Baptiste Coignard, 1694, entrée : couleur) : «Il se prend quelquefois plus estroitement pour une raison apparente dont on se sert pour couvrir et pallier quelque mensonge, ou quelque mauvaise action, afin de persuader ce qu'on desire. Couleur de Rhetorique. dans la Rhetorique on se sert de couleurs. "

11. J. J. Murphy observe que l'expression de «couleurs de rhétorique » est une nouveauté médiévale, mais que l'ensemble des procédés qu'elle recouvre n'est pas stable (J.J.Murphy, Rhetoric in the Middle Ages. A History of Rhetorical Theory from St. Augustine to the Renaissance, Berkeley/Los Angeles/London, University of California Press, 1974, p. 189-190). L'usage que les hommes de la Renaissance font de "couleurs» semble indiquer un sémantisme plus large, englobant l'ensemble des figures et tropes.

12. J. Thenaud, La Lignée de Saturne ou Le Traité de science poétique, éd. G. Mallary Masters, Genève, Droz, 1973, chap. X, p. 121.

13. J. Lemaire de Belges, La Légende des Vénitiens, op. cit., prologue, p. 5.

14. Ibid., péroraison, p. 37.

15. J. de Léry, Histoire d'un voyage fait en la terre du Brésil, op. cit., p. 34. 
16. J. Calvin, Institution de la religion chrestienne, mis en quatre livres et distinguee par chapitres, Genève, Jacques Bourgeois, 1562, livre I, chap. 8, p. 32.

17. R. Benoist, La Sainte Bible, Contenant le Vieil et Nouveau testament, Paris, Sébastien Nivelle, 1568, "Advertissements apologetiques ", $4^{\mathrm{e}}$ avertissement, n. p.

18. J. Molinet, Chroniques, éd. G. Doutrepont et O. Jodogne, Bruxelles, Palais des Académies, 1935, vol. 2, prologue (2e version), p. 593.

19. N. Du Fail, Propos rustiques, Lyon, Jean de Tournes, 1547, p. 13.

20. R. Estienne, Le Nouveau Testament, [Genève], Robert Estienne, 1552, cité par M.-C.GomezGéraud dans Histoire des traductions en langue française, op. cit., p. 474.

21. Montaigne, Essais, éd. J. Balsamo, M. Magnien, C. Magnien-Simonin, Paris, Gallimard «Bibliothèque de la Pléiade ", 2007, III, 12, p. 1101.

22. Cicéron, L'Orateur, éd. et trad. A. Yon, Paris, Les Belles Lettres, 1964, XXIII, § 78-79. Pour une analyse de ce passage, voir C. Noirot, "Entre deux airs». Style simple et ethos poétique chez Clément Marot et Joachim Du Bellay (1515-1560), Paris, Hermann, 2013, p. 51.

23. Cicéron, L'Orateur, op. cit., XXIV, § 81 et XXv, § 86. Voir C. Noirot, «Entre deux airs ", op. cit., p. 52.

24. Montaigne, Essais, op. cit., I, 51, p. 327.

25. J. Calvin, Des scandales, éd. O. Fatio, Genève, Droz, 1984, p. 65.

26. Ibid., p. 65-66. Voir aussi ce que Calvin dit dans l'Institution de la religion chrestienne (op. cit., I, 8, p. 28) : « puis que telle simplicité rude et quasi agreste nous esmeut en plus grande reverence que tout le beau langage des Rhetoriciens du monde, que pouvons-nous estimer, sinon que l'Escriture contient en soy telle vertu de verité, qu'elle n'a aucun besoin d'artifice de paroles?»

27. R. Benoist, La Sainte Bible, op. cit., «Advertissemens Apologetiques », $4^{\mathrm{e}}$ avertissement, n. p. Ce passage est commenté par M.-C. Gomez-Géraud qui y voit un «topos en vogue surtout dans la littérature protestante : celui de la vérité sans fard, servie par un style simple ", utilisé ici par un prédicateur catholique (Histoire des traductions en langue française, op. cit., p. 483).

28. C. Noirot, «Entre deux airs », op. cit., p. 67-88.

29. O. Millet, « Les préfaces aux traductions françaises de la Bible (1523-1588) : la question de la langue ", dans C. Bruckner dir., Traduction et adaptation en France, Paris, Champion, 1997, p. 373-387.

30. L. Susini, "Style simple et style figuré à l'âge classique : émergence d'une concordia discors ", dans B. Polduc et H. Goldwyn dir., Concordia discors. Choix de communications présentées lors du $41^{e}$ congrès annuel de la North American Society for Seventeenth-Century French Literature, New York University, 20-23 mai 2009, Tübingen, Narr Verlag, 2009, p. 65-66: «D'un bout à l'autre du XVII siècle, en somme, sans grande originalité mais de manière extrêmement massive, le style figuré s'oppose au style simple comme l'artifice à la nature, voire, parfois, comme les mots aux choses, ou comme le faux au vrai - sans exclusion du fait que les puissances d'erreur puissent bien souvent valoir chemin de vérité. »

31. Quintilien, Institution oratoire, IX, 1, 4, éd. et trad. J. Cousin, Paris, Les Belles Lettres, 1978.

32. F. Rabelais, Gargantua, éd. M. Huchon, Paris, Gallimard, «Folio classique », 2007, chap. 39, p. 361.

33. Sur l'affinité des notions de figure et de fiction sous l'Ancien régime, voir S. Duval et A. Petit, "Que veut dire fiction sous l'Ancien Régime? ", Littératures classiques, n 96, 2018, p. 41-52.

34. Montaigne, Essais, op. cit., III, 11, p. 1074.

35. M. McGowan, «L'hyperbole dans les Essais », dans J. O’Brien, M. Quainton et J. J. Supple dir., Montaigne et la rhétorique, Paris, Honoré Champion, 1995, p. 99-114.

36. J. Molinet, Chroniques, op. cit., vol. 2, prologue ( $2^{\mathrm{e}}$ version), p. 593.

37. Ibid., vol. 3, p. 164 sq.

38. J. Peletier, Art poétique, chap. $\mathrm{X}$ "Des ornements de poésie", dans Traités de poétique et de rhétorique de la Renaissance, éd. F. Goyet, Paris, Livre de poche, 1990, p. 253. 
39. M.-H. Prat, « Métaphores et métonymies dans l'Histoire universelle : la fiction pour l'histoire », dans O. Pot dir., Entre Clio et Melpomène. Les fictions de l'histoire chez Agrippa d'Aubigné, Paris, Classiques Garnier, 2010, p. 111-143.

40. J. Lemaire de Belges, La Legende des Venitiens, op. cit., p. 19.

41. Ibid., p. 32.

42. Ibid., péroraison, p. 38.

43. Ibid., respectivement p. 26 et p. 32 .

44. Ibid., p. 37.

45. Id., Traicté de la différence des schismes et des conciles de l'Église, éd. J. Britnell, Genève, Droz, 1997, p. 138.

46. Ibid., p. 83 : « ... comme le triacle et mytridat sont ennemyz de poisons et venins »; p. 115 : « laquelle [couronne des papes] est haulte et poinctue, comme une coqueluche, et riche comme la boitte d'un grand lapidaire oriental »

47. Ibid., p. 195. Voir aussi, p. 84 l'emploi du proverbe «car tousjours pourceaulx paistront glands ».

48. A. Desbois-Ientile, Lemaire de Belges, Lemaire Belgeois, Paris, Classiques Garnier, 2019, p. 601-653.

49. Le trope peut présenter une fonction cognitive comme nous l'avons montré dans un article («Métaphores et savoir dans l'œuvre de Jean Lemaire de Belges», dans B. Petey-Girard et C. Trotot dir., Métaphore, savoirs et arts au début des temps modernes, Paris, Classiques Garnier, 2015, p. 299-316), mais ce qui nous paraît symptomatique est le fait que Vénus s'exprime par métaphores et non par des mots de sens propre.

50. J. Lemaire de Belges, Le Temple d'honneur et de vertus, Genève/Paris, Droz/Minard, 1957, p. 44 : «[...] affin que vous veissiez voz crys dedans escriptz en couleur de douleur, plains de tous plaintz, et que voz soulas qui sont las et voz rys qui sont perilz prinssent quelque sourse de ressourse ».

51. Sur ces enjeux, voir F. Cornilliat, "Or ne mens ». Couleurs de l'Éloge et du Blâme chez les "Grands rhétoriqueurs ", Paris, Champion, 1994.

52. J. Lemaire, Les Illustrations de Gaule et singularitez de Troye, I, 32, dans Euvres, éd. Stecher, Louvain, Lefever, 1882, vol. I, p. 249. R. M. Berrong voit dans Lemaire un auteur conscient des dangers de l'éloquence, capable de ravir l'auditeur et le déposséder de sa capacité à raisonner ("Les Illustrations de Gaule et Singularitez de Troye: Jean Lemaire de Belges' Ambivalent View of "Eloquence" ", Studi francesi, n 78, 1982, p. 399-407).

53. Voir notre article "De Vénus à Minerve : variations fictionnelles dans La Concorde des deux langages ", à paraître dans les Mélanges en l'honneur de Mireille Huchon.

54. O. Millet, Calvin et la dynamique de la parole. Étude de rhétorique réformée, Paris, Champion, 1992, p. 419 : « la présence de l'ornement, pour autant qu'il relèverait soit de la virtuosité du genre démonstratif, soit d'une esthétique de la mimésis, autrement dit de la fiction poétique, est systématiquement nié par l'exégète, et expliqué et justifié dans une autre perspective ».

55. Ibid., p. 421. 


\section{AUTEUR}

\section{ADELINE DESBOIS-IENTILE}

Sorbonne Université - EA 4509 STIH 